\title{
Isoliquiritigenin inhibits the proliferation and induces the differentiation of human glioma stem cells
}

\author{
YULIANG LIN*, HONGJUN SUN**, YING DANG and ZHIYUN LI \\ Department of Neurosurgery, Lanzhou General Hospital, Lanzhou Command of CPLA, Lanzhou, Gansu 730050, P.R. China
}

Received June 8, 2017; Accepted November 16, 2017

DOI: $10.3892 /$ or.2017.6154

\begin{abstract}
Glioma stem cells (GSCs) have been proven to be resistant to various therapeutic strategies, such as temozolomide chemotherapy and radiotherapy, leading to glioma recurrence. Isoliquiritigenin (ISL), a menber of the flavonoids isolated from liquorice has been found to be a potent stimulator of cell differentiation and has potential application for treating various types of cancer including human brain glioma. However, the antitumor activity of ISL on GSCs and the signaling pathway underlying its therapeutic effects are poorly understood. In the present study, GSCs were isolated from SHG44 human glioma cells by serum-free culture and treated with ISL or DAPT (a Notch $/ \gamma$-secretase inhibitor). It was found that ISL dose-dependently inhibited GSC growth after $72 \mathrm{~h}$ of treatment and decreased the formation of tumor spheres. Meanwhile, GSCs differentiated into astrocytes and neurons. Furthermore, these therapeutic effects were accompanied by downregulation of Notch1 and Hes1 at the protein and mRNA levels. Taken together, our results demonstrated that ISL exhibits antitumor effects on GSCs by inhibiting proliferation and inducing differentiation. The therapeutic effect may be related to downregulation of the Notch1 signaling pathway. Application of ISL presents potential benefits for the treatment of human brain glioma.
\end{abstract}

\section{Introduction}

Eighty percent of malignant tumors that develop in the central nervous system (CNS) are malignant gliomas, which display histological similarities to glial cells, including astrocytes and oligodendrocytes (1). Glioblastoma multiforme (GBM) is the most common primary malignant adult brain tumor. GBM patients have a median survival of 13-16 months even

Correspondence to: Professor Zhiyun Li, Department of Neurosurgery, Lanzhou General Hospital, Lanzhou Command of CPLA, 333 South Binhe Road, Qilihe, Lanzhou, Gansu 730050, P.R. China E-mail: lizhiyun654@163.com

${ }^{*}$ Contributed equally

Key words: isoliquiritigenin, proliferation, differentiation, Notch, glioma stem cells following therapy (2). The current standard of care consists of maximal safe surgical resection with a combination radiotherapy and adjuvant temozolomide chemotherapy. However, the patient 5-year survival rate is $<10 \%$ (3). Malignant gliomas are aggressive brain tumors with limited therapeutic options, possibly because of the highly tumorigenic subpopulations of glioma stem cells (GSCs) (4). GSCs have been proven to be resistant to various chemotherapeutic agents, such as temozolomide, the first-line treatment for glioblastoma, allowing these cells to survive therapy, leading to radioresistance and tumor relapse $(5,6)$. As the current treatment modalities of GBM are not specifically designed to kill GSCs and the effectiveness is limited, new agents targeting GSCs for potential use in GBM clinical therapy have attracted attention.

Isoliquiritigenin (ISL), extracted from Glycyrrhiza spp., is a promising candidate as a natural, alternative method of cancer therapy (7). It is a flavonoid compound with efficient antitumor activity (8). In particular, many studies both in vitro and in vivo, have demonstrated the antitumor efficacy of ISL against various types of cancer (9-12). It has been suggested that ISL can inhibit proliferation and induce apoptotic and necrotic cell death of glioma cells (13). In the present study, the antitumor activity of ISL on human GSCs was examined.

Notch signaling has been reported to be involved in fetal development and tissue homeostasis by directing many cellular functions, including cell growth and differentiation, cell fate determination and regulation of stem cell maintenance (14). Notch signaling pathway is an attractive target for cancer therapy as targeting Notch signaling could overcome multi-drug resistance (MDR) (15). It was found that a Notch/ $\gamma$-secretase inhibitor (GSI) in combination with radiotherapy and temozolomide attenuated proliferation, decreased glioma stem cell markers and resulted in a marked reduction in clonogenic survival in primary and established glioma cell lines (16). Here, we hypothesized that the anticancer effects of ISL are related to the Notch1 signaling pathway. The present study proposes the possibility of the therapeutic application and an intracellular antitumor mechanism of ISL in GSC-targeted treatment for human brain glioma.

\section{Materials and methods}

Isolation of GSCs. SHG44 human glioma cells from the American Type Culture Collection (ATCC, Manassas, VA, USA) were cultured in Dulbecco's modified Eagle's medium 
(DMEM) supplemented with $10 \%$ fetal bovine serum (FBS), $100 \mathrm{U} / \mathrm{ml}$ penicillin and $100 \mu \mathrm{g} / \mathrm{ml}$ streptomycin at $37^{\circ} \mathrm{C}$ in a humidified incubator with $5 \% \mathrm{CO}_{2}$. Tumor cells grown to $80 \%$ confluence were rinsed with sterile phosphate-buffered saline (PBS) $(0.01 \mathrm{M}, \mathrm{pH} 7.4)$ and digested at $37^{\circ} \mathrm{C}$ with $0.25 \%$ trypsin for $1 \mathrm{~min}$, then resuspended in a serum-free neural stem cell medium consisting of D-MEM/F-12, 2\% B27 (Gibco BRL, Gaithersburg, MD, USA), 20 ng/ml EGF (PeproTech, Inc., Rocky Hill, NJ, USA), 20 ng/ml bFGF (PeproTech, Inc.), and then seeded into 6-well suspension cell culture plates (Beaver, China). After 7 days of culture at $37^{\circ} \mathrm{C}$ in $5 \%$ humidified $\mathrm{CO}_{2}$ atmosphere, the medium was removed by centrifugation and GSC-forming spheres were digested with $0.25 \%$ trypsin into a single-cell suspension. GSCs were subcultured and enriched three times before being used for assays.

Identification of surface phenotypes of GSCs. GSCs grown on polylysine-coated coverslips were fixed using $4 \%$ paraformaldehyde for $15 \mathrm{~min}$ and permeabilized with $0.5 \%$ Triton X-100 (PBST) for $10 \mathrm{~min}$. The coverslips were blocked with $5 \%$ bovine serum albumin (BSA) in PBS $(0.01 \mathrm{M}, \mathrm{pH} 7.4)$ for $30 \mathrm{~min}$ at room temperature, followed by incubation with primary antibodies targeted against rabbit anti-CD133 (\#PAB12663; 1:200; Abnova), mouse anti-nestin (\#ab18102; 1:200; Abcam) and rabbit anti-Bcl-2 (\#ab32124; 1:100; Abcam) overnight at $4^{\circ} \mathrm{C}$. After three washes with PBS, the coverslips were incubated with PE-labeled rabbit anti-mouse $\operatorname{IgG}$ (\#ab7000; 1:100; Abcam) and FITC-labeled goat anti-rabbit IgG (\#ab6717; 1:1,000; Abcam) for 120 min at $37^{\circ} \mathrm{C}$. Nuclear DNA was labeled in blue with DAPI. The staining results were imaged on a Nikon C-1 laser-scanning confocal microscope (Nikon, Tokyo, Japan).

Treatments. After the cells were grown to sub-confluence, they were supplemented with various concentrations $(10,20,40$, 80 and $160 \mu \mathrm{M}$, respectively) of ISL (purity $\geq 98 \%$; Shanghai Yuanye Bio-Technology Corp., Shanghai, China), $2.5 \mu \mathrm{M}$ DAPT (a Notch $/ \gamma$-secretase inhibitor) (purity $>98.5 \%$; Gene Operation, USA) or 10\% FBS (as positive control) for different times. Treatment effects on proliferation and differentiation were examined by further assays described below.

Cell proliferation assay. Cell Counting Kit-8 (CCK-8; Dojindo, Kumamoto, Japan) assay was used to measure cell proliferation of the GSCs. Briefly, $3 \times 10^{3}$ cells per well were seeded into 96-well suspension cell culture plates (Beaver) in triplicate. At the appropriate time (12, 24, 48 and $72 \mathrm{~h}), 10 \mu \mathrm{l}$ CCK-8 solution was added to each well, and the plates were incubated for $4 \mathrm{~h}$ at $37^{\circ} \mathrm{C}$ under a moist atmosphere with $5 \%$ $\mathrm{CO}_{2}$. The absorbance at $450 \mathrm{~nm}$ was measured using the Gen5 microplate reader (BioTek, Winooski, VT, USA).

The formation of GSC spheres was observed and imaged after 9 days of treatment (ISL was supplemented every other day) using an Olympus IX71 Inverted Microscope (Olympus, Tokyo, Japan). The diameters and numbers of GSC-forming spheres were calculated from 5 random fields of view by Image-Pro Plus 6.0 software.

Immunofluorescence staining. After $48 \mathrm{~h}$ of treatment, GSCs grown on polylysine-coated coverslips were fixed in
4\% paraformaldehyde for $15 \mathrm{~min}$ and permeabilized with $0.5 \%$ Triton X-100 (PBST) for $10 \mathrm{~min}$. The coverslips were blocked with 5\% BSA in PBS $(0.01 \mathrm{M}, \mathrm{pH} 7.4)$ for $30 \mathrm{~min}$ at room temperature, followed by incubation with primary antibodies targeted against mouse anti-GFAP (\#ab4648; 1:50; Abcam), mouse anti- $\beta$ III Tubulin (\#ab78078; $1: 1,000$; Abcam) and rabbit anti-Hes1 (\#ab119776; 1:100; Abcam) overnight at $4^{\circ} \mathrm{C}$. After three washes with PBS, the coverslips were incubated with PE-labeled rabbit anti-mouse IgG (\#ab7000; 1:100; Abcam) and FITC-labeled goat anti-rabbit IgG (\#ab6717; 1:1000; Abcam) for $120 \mathrm{~min}$ at $37^{\circ} \mathrm{C}$. Nuclear DNA was labeled in blue with DAPI. The fluorescent sections were observed and photographed with a Nikon C-1 laser-scanning confocal microscope (Nikon). The number of GFAP ${ }^{+}$cells and $\beta$ III tubulin ${ }^{+}$cells were determined in 5 random fields of view by Image-Pro Plus 6.0.

Quantitative real-time PCR ( $q P C R)$. The cultured cells were harvested and lysed at $48 \mathrm{~h}$ after ISL and DAPT treatment. Total cellular RNA was extracted using TRIzol reagent (Takara Biotechnology Co., Ltd., Dalian, China). To remove any DNA contamination, RNA samples were treated with Recombinant DNase I (Takara Biotechnology). Single-stranded cDNA was synthesized from total RNA with the PrimeScript ${ }^{\mathrm{TM}}$ RT Master Mix (Takara Biotechnology). qPCR was performed for HES1 and internal control GAPDH using the SYBR Premix Ex Taq ${ }^{\mathrm{TM}}$ II (Takara, Shiga, Japan) on a 7300 real-time PCR system (Applied Biosystems, Singapore, Singapore). All reactions were carried out in triplicate and expression data (after being calibrated with GAPDH levels) were analyzed using the $2^{-\triangle \Delta C a}$ method. Optimal oligonucleotide primers used in the above PCR assays were designed and synthesized by Takara Biotechnology based on published human cDNA sequences. The primer sequences were as follows: HES1 sense 5'-GTGT CAACACGACACCGGATAAAC-3' and antisense 5'-CAGA ATGTCCGCCTTCTCCAG-3'; GAPDH sense 5'-GCACCGT CAAGGCTGAGAAC-3' and antisense 5'-TGGTGAAGAC GCCAGTGGA-3'. All reactions were run in triplicate.

Western blot analysis. The protein expression levels of differentiation markers (GFAP and $\beta$ III tubulin) and Notch1 signaling pathway markers (Notch1 and Hes1) were examined by western blotting. $\beta$-actin levels were evaluated as a loading control. Briefly, cells were lysed and homogenized in RIPA lysis buffer (Solarbio, Beijing, China). The supernatants were obtained by centrifugation at $4^{\circ} \mathrm{C}$ for $10 \mathrm{~min}$ at $12,000 \mathrm{rpm}$ and the total protein contents were quantified using a BCA kit (Solarbio). For electrophoresis, equal amounts of protein ( $15 \mu \mathrm{g} /$ lane) were separated by sodium dodecyl sulfate-polyacrylamide gel electrophoresis (SDS-PAGE) analysis on 8, 10 or $12 \%$ polyacrylamide gel depending on the target protein and transferred onto PVDF membranes (Millipore, Billerica, MA, USA) using a wet electroblotting system (Mini Trans-Blot; Bio-Rad). For immunoblotting, the membranes were blocked in $5 \%$ non-fat dry milk for $2 \mathrm{~h}$ at room temperature and incubated overnight at $4{ }^{\circ} \mathrm{C}$ with one of the following primary antibodies: GFAP (\#ab4648; 1:400; Abcam), $\beta$ III tubulin (\#ab78078; 1:800; Abcam), Notch1 (\#ab128076; 1:800; Abcam), Hes1 (\#ab119776; 1:800; Abcam) and $\beta$-actin (\#AP0060; 1:3,000; Bioworld Technology Inc.). After three washes in Tris-buffered 

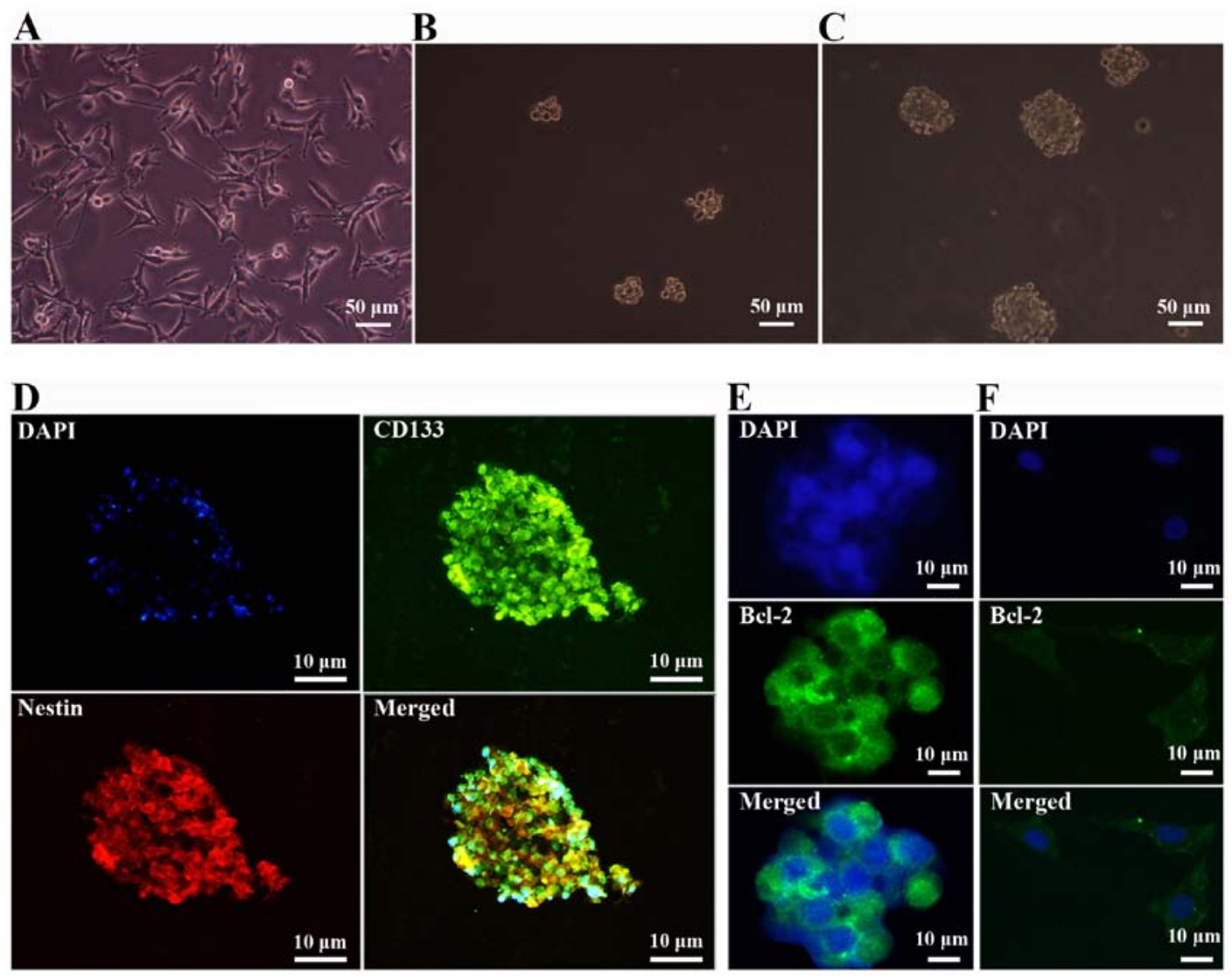

Figure 1. Micrographs of GSCs and the phenotypic characterization. (A) Glioma cells showed adherent growth. Representative images of neurospheres formed by SHG44 GSCs 4 days (B) and 7 days (C) after plating in serum-free medium; scale bar, $50 \mu \mathrm{m}$. (D) Neurosphere-like colonies were positive for CD133 (green) and Nestin (red), and the nuclei were stained with DAPI (blue); scale bar, $50 \mu \mathrm{m}$. Tumor spheres (E) and SHG44 human glioma cells (F) were stained for Bcl-2 proto-oncogene (green); scale bar, $10 \mu \mathrm{m}$. GSCs, glioma stem cells.

saline containing $0.5 \%$ Tween-20 (TBST), the samples were treated with HRP-conjugated goat anti-rabbit (\#E030120-01; 1:10,000; EarthOx Life Science, Millbrae, CA, USA) or goat anti-mouse secondary antibodies (\#ab6728; 1:10,000; Abcam) for $1 \mathrm{~h}$ at room temperature. The antibody labeling was visualized using enhanced chemiluminescence reagent (Millipore) and exposed on X-ray film (Kodak, China). For the immunoblot analyses, densitometry was performed with Image-Pro Plus 6.0 software. The optical densities (ODs) of the protein bands were calculated relative to the ODs of the reference protein $\beta$-actin.

Statistical analysis. Data are expressed as the mean \pm SD. All statistical analyses were performed using the SPSS software program (version 21.0; IBM Corp., Armonk, NY, USA). The differences between the experimental groups were analyzed by one-way analysis of variance (ANOVA). $\mathrm{P}<0.05$ was considered statistically significant.

\section{Results}

Glioma cells and GSC-forming spheres. Within 48-72 h of serum-free culture SHG44 human glioma cells yielded a minority fraction of cells that possessed extensive proliferative and self-renewal potential, and generated free-floating neurosphere-like brain tumor spheres. These spheres gradually enlarged as the time of the culture increased (Fig. 1B and C).
In comparison, glioma cells cultured in DMEM with 10\% FBS growed adherently (Fig. 1A).

Characterization of surface phenotypes of GSCs. Expression of GSC surface markers were examined in human GSC-forming spheres. As shown in Fig. 1D, the cells expressed characteristic neural stem cell (NSC) markers CD133 and Nestin. SHG44 human glioma cells and tumor neurospheres were postive for the proto-oncogene $\mathrm{Bcl}-2$ which distinguished them from normal neurons and NSCs (Fig. 1E and F). The immunofluorescence staining data indicated that the tumor spheres expressed typical surface markers of GSCs and thus were used for experiments as described below.

ISL inhibits cell proliferation in a dose-dependent manner. To investigate the influence of ISL on GSC proliferation, we firstly examined the activities of cells treated with various concentrations of ISL for different times. As shown in Fig. 2A, within 24 and $48 \mathrm{~h}$ of treatment the proliferative activity of the cells was significantly higher in the 10, 20 and $40 \mu \mathrm{M}$ ISL groups than that noted in the $0 \mu \mathrm{M}$ ISL group. There was no significant difference between the 0 and $80 \mu \mathrm{M}$ ISL group within $24 \mathrm{~h}$, but the activity of cells began to decline after $48 \mathrm{~h}$. After $72 \mathrm{~h}$, the cell proliferation of the ISL treatment groups were obviously inhibited in a dose-dependent manner, with a half inhibitory concentration $\mathrm{IC}_{50}$ of $102.744 \mu \mathrm{mol} / \mathrm{l}$. 


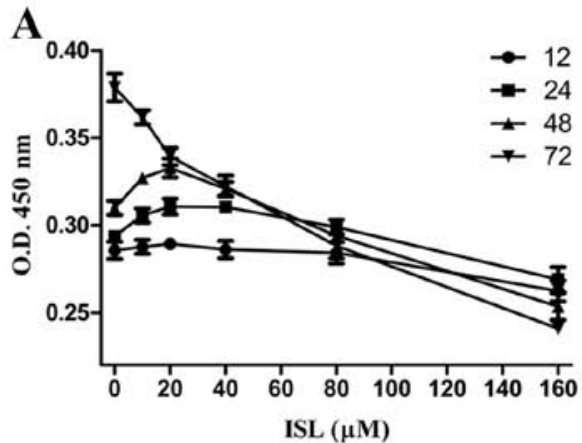

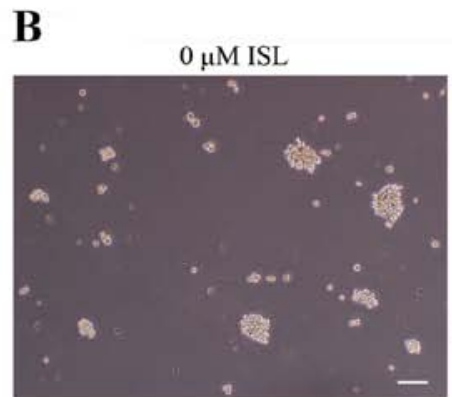
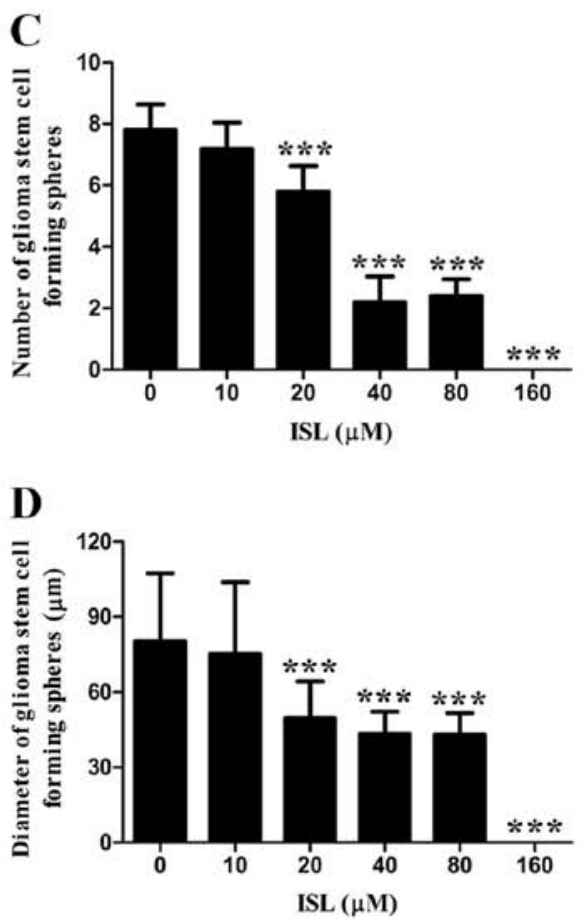

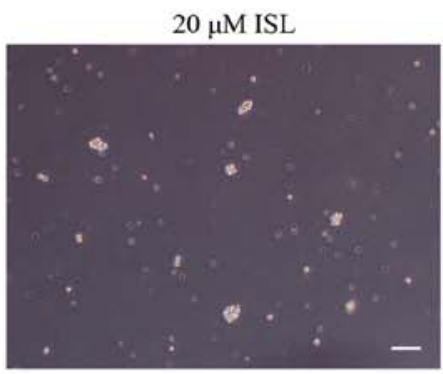

$80 \mu \mathrm{M}$ ISL

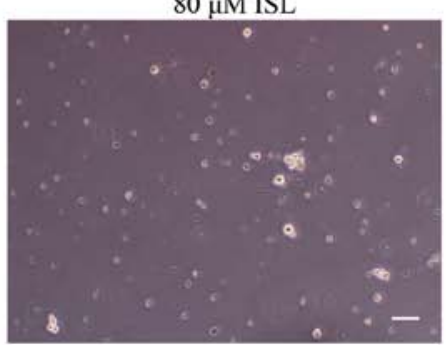

$10 \mu \mathrm{M}$ ISL

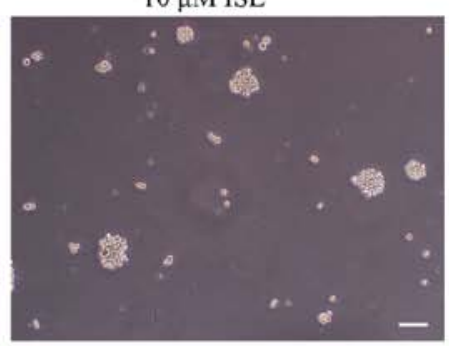

$40 \mu \mathrm{M}$ ISL

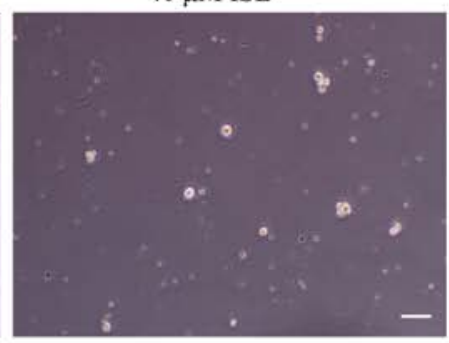

$160 \mu \mathrm{M}$ ISL

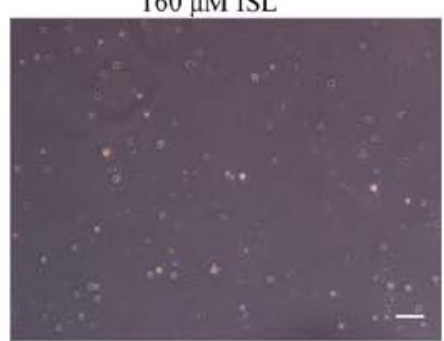

Figure 2. Growth inhibitory effect of ISL on GSCs. (A) Cell proliferative activity was determined at 12, 24, 48 and $72 \mathrm{~h}$ after ISL (0, 10, 20, 40, 80 and $160 \mu \mathrm{M}$ ) treatment by CCK-8 assay. Data were summarized from 3 independent repeated experiments as mean \pm SD. (B) Representative images of tumor spheres 9 days after ISL treatment; scale bar, $100 \mu \mathrm{m}$. (C and D) The numbers and diameters of GSC-forming spheres on the 9th day of ISL intervention were measured by Image-Pro Plus 6.0. Data were summarized from 5 random fields of view as mean \pm SD. ${ }^{* * * *} \mathrm{P}<0.001$ vs. the $0 \mu \mathrm{M}$ ISL. GSCs, glioma stem cells. ISL, isoliquiritigenin.

Formation of neurospheres is a morphological feature of GSCs. Then we examined the influence of ISL on GSC-forming spheres on the 9th day of treatment. It was found that the $0 \mu \mathrm{M}$ ISL group had the largest diameter and highest number of tumor spheres formed from the GSCs (Fig. 2B-D). The diameters and the numbers were both decreased by ISL in a dose-dependent manner, and $160 \mu \mathrm{M}$ ISL completely prevented neurophere formation, displaying a similar tendency with cell activity at $72 \mathrm{~h}$.

Differentiation-promoting action of ISL on GSCs. To determine whether ISL promotes the differentiation of GSCs, intracellular differentiation markers GFAP and $\beta$ III tubulin were examined after $48 \mathrm{~h}$ of ISL treatment by immunofluorescence staining. As shown in Fig. 3, the numbers of differentiation marker-positive cells were higher in the ISL group and DAPT group than that in the control group after $48 \mathrm{~h}$. As a comparison, FBS effectively induced the differentiation of GSCs.
To determine whether ISL inhibits Hes1 (Notch1 target gene) in the differentiated cells, GFAP and Hes1 were assessed together after the cells were treated for $48 \mathrm{~h}$. The relative intensity of Hes1 was decreased in the $\mathrm{GFAP}^{+}$cells induced by ISL (Fig. 4).

The protein expression levels of differentiation markers were also examined after $48 \mathrm{~h}$ of ISL treatment. As expected, the protein expression levels of GFAP and $\beta$ III tubulin in the DAPT group and ISL groups were higher than those of the control, and DAPT induced higher GFAP and $\beta$ III tubulin protein expression levels compared to the ISL groups. However, there was no obvious difference between the low dose $(20 \mu \mathrm{M})$ and high dose ( $80 \mu \mathrm{M})$ ISL groups (Fig. 5A and B).

Ability of ISL to downregulate the Notch signaling pathway. To examine whether ISL inhibits the proliferation and induces the differentiation of human GSCs through Notch1 signaling, Notch1 and Hes1 were analyzed $48 \mathrm{~h}$ after GSCs were respectively treated with ISL and DAPT. The predicted molecular 
A

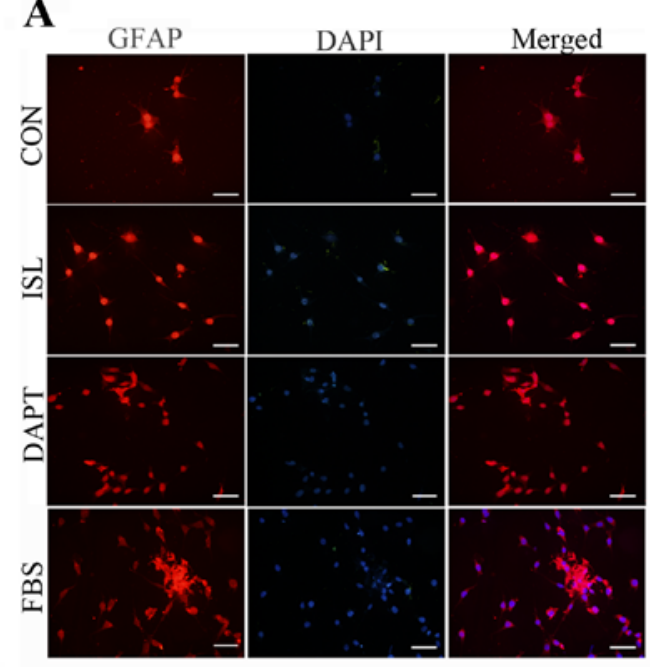

C

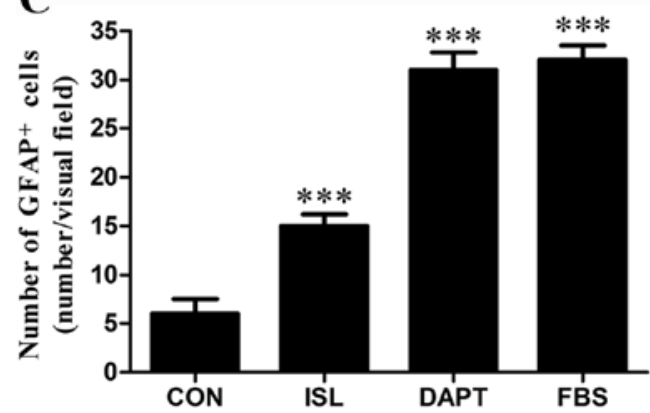

B

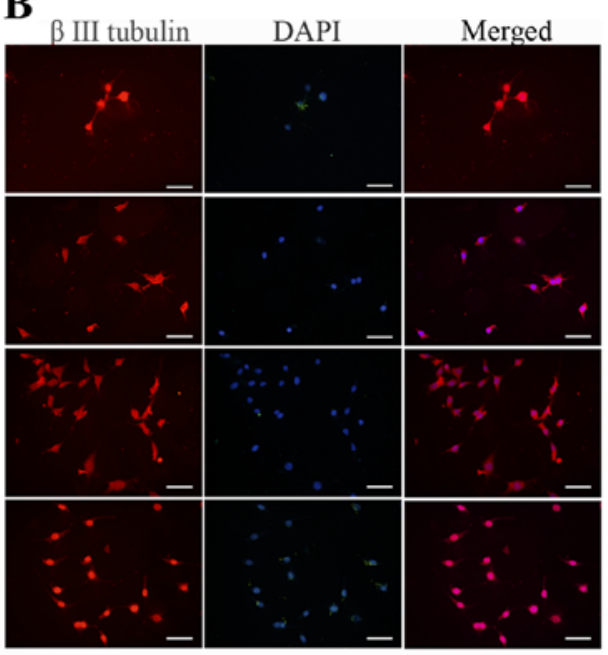

D

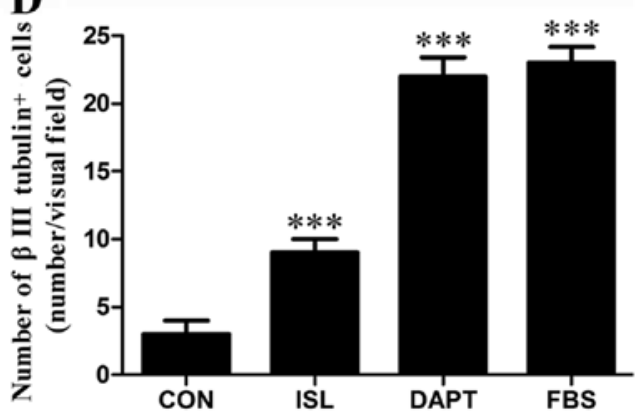

Figure 3. Differentiation-inducing effect of ISL $(20 \mu \mathrm{M})$ on GSCs. The intracellular expression of differentiation markers at $48 \mathrm{~h}$ was examined by immunofluorescence staining. GFAP (A) and $\beta$ III tubulin (B) were assessed by staining with PE-labeled rabbit anti-mouse IgG (red), and the nuclei were stained with DAPI (blue); scale bar, $50 \mu \mathrm{m}$. (C and D) The number of GFAP ${ }^{+}$and $\beta$ III tubulin ${ }^{+}$cells per visual field were determined from 5 random fields of view by Image-Pro Plus 6.0. Data are summarized as mean $\pm \mathrm{SD} .{ }^{* * *} \mathrm{P}<0.001$ vs the control (CON). GSCs, glioma stem cells. ISL, isoliquiritigenin.

A

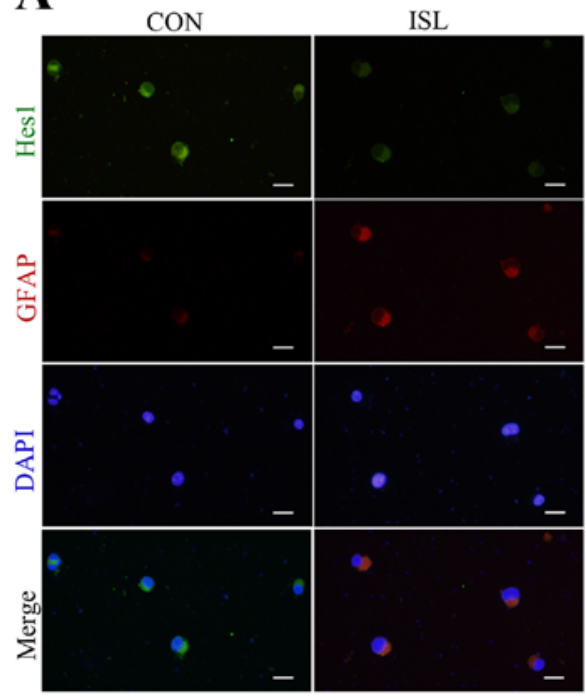

B
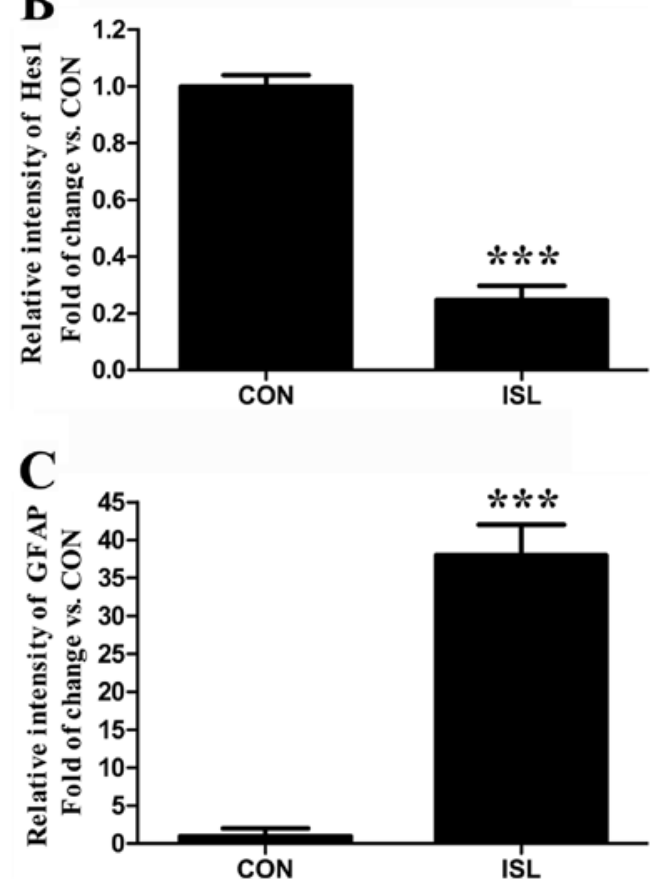

Figure 4. A decrease in Hes1 is noted in differentiated cells induced by ISL (20 $\mu \mathrm{M})$. (A) The intracellular expression of Hes1 and GFAP was examined by immunofluorescence staining. Hes1 was assessed by staining with FITC-labeled goat anti-rabbit IgG (green), GFAP was assessed by staining with PE-labeled rabbit anti-mouse IgG (red), and the nuclei were stained with DAPI (blue); scale bar, $50 \mu \mathrm{m}$. The relative intensity of Hes1 (B) and GFAP (C) in the cells was measured in 5 random fields of view by Image-Pro Plus 6.0 . Data are expressed as mean \pm SD. ${ }^{* * *} \mathrm{P}<0.001 \mathrm{vs}$. the control (CON). ISL, isoliquiritigenin. 
A
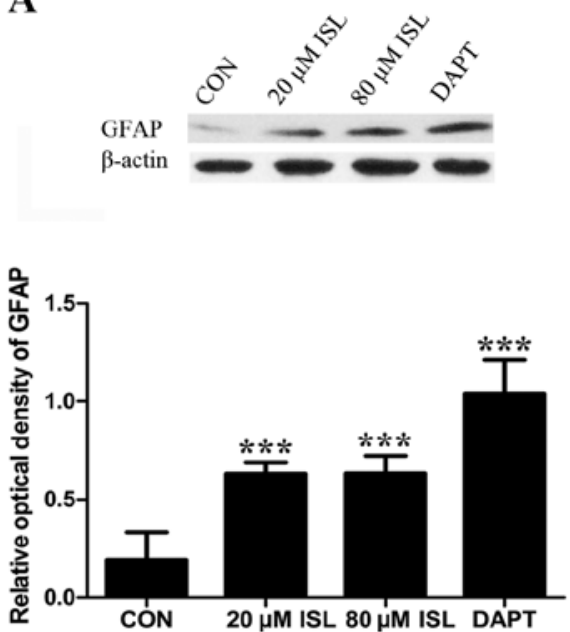

B
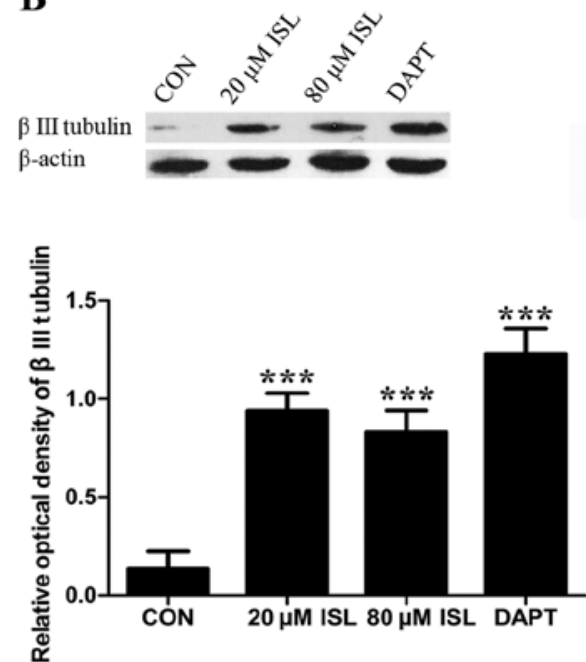

C
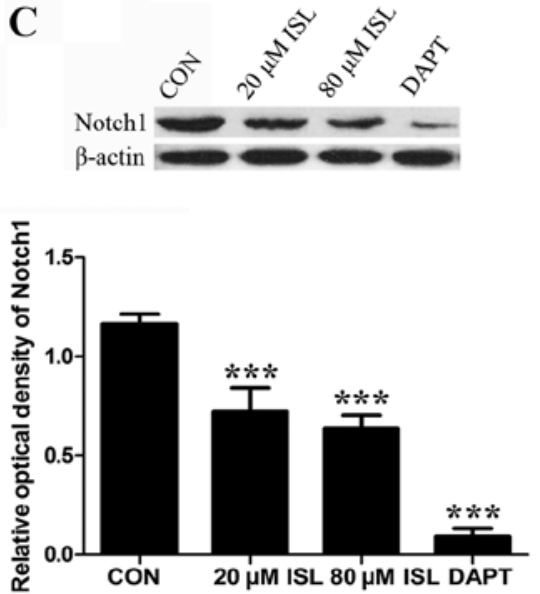

D
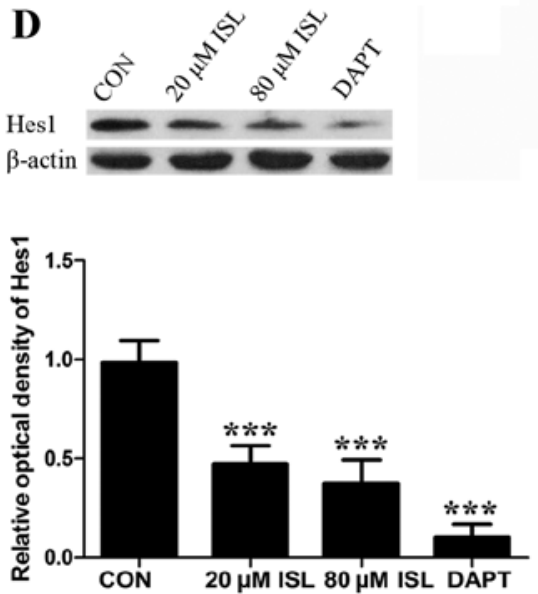

$\mathbf{E}$

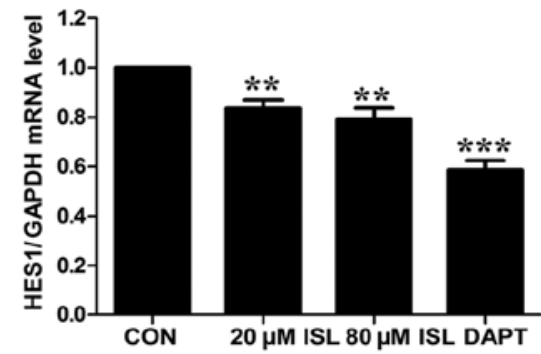

Figure 5. Effect of ISL treatment on differentiation markers and Notch1 signaling pathway in GSCs. The protein expression levels of GFAP (A) and $\beta$ III tubulin (B) were examined after the cells were treated for $48 \mathrm{~h}$. The protein expression level of Notch $(\mathrm{C})$ and the mRNA and protein expression level of Hes1 (D and E) were examined at $48 \mathrm{~h}$ after ISL treatment. The band observed at $120 \mathrm{kDa}$ could potentially be a truncated form of Notch1, following cleavage by furin-like protease. For the immunoblotting analyses, densitometry was performed with Image-Pro Plus 6.0 software. The optical densities (ODs) of the protein bands were calculated relative to the ODs of the reference protein $\beta$-actin. Data are summarized as mean $\pm \mathrm{SD}{ }^{* * *} \mathrm{P}<0.01,{ }^{* * * *} \mathrm{P}<0.001$ vs. the control (CON). GSCs, glioma stem cells. ISL, isoliquiritigenin.

weight of Notch1 is $272 \mathrm{kDa}$. In the present study, the band observed at $120 \mathrm{kDa}$ could potentially be a truncated form of Notch1, following cleavage by furin-like protease. The protein expression levels of Notch1 in the DAPT group and ISL groups were lower than those of the control (Fig. 5C). The mRNA and protein expression levels of Hes1 were also significantly decreased compared to the control (Fig. 5D and E). DAPT induced lower Notch1 and Hes1 expression levels compared to the ISL groups.

\section{Discussion}

It has been reported that supplementation with ISL may be an effective approach to inhibit the growth of tumors and counteract the adverse effects of existing drugs for cancer patients (17). Despite the studies demonstrating the efficacy of ISL in tumor therapy, its anticancer activities against GSCs remain unclear. In the present study, we identified that ISL inhibited proliferation and induced differentiation of human GSCs, and inhibited the activation of the Notch1 pathway.

Serum-free culture is a simple and convenient method for neural stem cell (NSC) enrichment and has been applied for cancer stem cell (CSC) isolation from brain tumors and many other types of cancers (18). Using serum-free medium with supplemental factors B27, EGF and bFGF, the present study isolated tumor spheres from SHG44 human glioma cells on suspension cell culture plates. CD133 and Nestin are important cell surface markers present on NSCs. A high level of CD133 expression may be an independent risk factor for glioma patient prognosis and high expression of Nestin tends to correlate with a worse outcome for glioma patients (19). As previously described, GSCs develop high chemoresistance and radioresistance. In this respect, a high level of CD133 or/and Nestin expression in glioma cells may imply a high proportion of GSCs which contribute to adverse prognosis. In this study, CD133 and Nestin were used together to examine the stem 
cell property of tumor spheres and the expression of Bcl-2 provided further evidence that these neurospheres were GCSs derived from the SHG44 cell line.

It has been reported that the inhibition rate of U87 human glioma cells can be $\sim 80 \%$ after a 72 -h treatment with 40-60 $\mu \mathrm{M}$ ISL $(13,20)$. Our data indicated that ISL can dose-dependently inhibit the proliferation of human SHG44 GSCs $72 \mathrm{~h}$ after treatment. GSC proliferation was obviously inhibited after a 72-h incubation with $10 \mu \mathrm{M}$ ISL. However, the $\mathrm{IC}_{50}$ of $102.744 \mu \mathrm{mol} / 1$ seemed much higher than that of other studies mentioned above. This might be because GSCs are proven to be more resistant to various chemotherapeutic agents. Some recent studies have suggested the use of ISL as a natural candidate to reduce CSC-like populations and colony formation, accompanied by enhanced CSC chemosensitivity $(21,22)$. Here, we investigated the inhibitory effect of ISL on the formation of glioma neurospheres for the first time. In terms of long-term efficacy, the diameters and the numbers of GSC-forming spheres on the 9th day of $20 \mu \mathrm{M}$ ISL treatment were significantly decreased. Thus, we believe that $20 \mu \mathrm{M}$ ISL can be sufficient to inhibit GSCs. Unfortunately, there are few toxicological evaluations reported for ISL treatment. Our findings will provide the theoretical and experimental bases for ISL clinical use in the treatment of glioma. Further studies are still required to investigate the clinical side-effects in vivo.

Here, we also showed the stimulating effect of ISL on GSC differentiation into downstream neural lineages. It has been reported that ISL facilitated the differentiation of human promyelocytic leukemia and mouse melanoma cells $(23,24)$. The present study first showed that ISL induced GSCs to differentiate into astrocytes (GFAP-positive) (25) and neurons ( $\beta$ III tubulin-positive) (26). As a result, after differentiation induced by ISL, the majority of GSCs were able to be killed by ISL, leading to decreased cell numbers. Neurons are highly differentiated cells and rich in tubulin isotypes (27). Another study found that $\beta$-tubulin is required for cell viability and neurite outgrowth, and the suppression of $\beta$ I isotype resulted in significant loss of cell viability during cell differentiation (28). In the present study, it is perhaps not coincidental that ISL increased the viability of GSCs at 24 and $48 \mathrm{~h}$ when a proliferation inhibitory effect was not dominant. Therefore, GSC proliferation was activated following $20 \mu \mathrm{M}$ ISL at $48 \mathrm{~h}$ when GSC viability was mainly increased in the differentiating cells. On the contrary, GSC proliferation was inhibited by $80 \mu \mathrm{M}$ ISL at $48 \mathrm{~h}$ as a high dose of ISL exhibited a strong growth inhibitory effect which abrogated the increase in viability of the differentiated cells. GSCs and NSCs express similar signatures, such as infinite self-renewal ability, neurosphere formation, and multipotential differentiation capacity $(6,29)$. GSCs in the control group treated without ISL still generated a small population of differentiated cells, but were much less than those in the ISL groups. Further studies are required to investigate the precise mechanism of how ISL induces GSCs to differentiate into each phenotype.

Recently, an accumulating body of evidence has emerged to suggest that the Notch1 signaling pathway plays an important role in cell fate decisions, and functions as a key regulator of cell growth, differentiation and proliferation (30,31). Fan et al (32) observed that inhibition of the Notch1 signaling pathway by a $\gamma$-secretase inhibitor resulted in the apoptosis and differentiation of medulloblastoma cell lines, and reduced tumor progression. In the present study, the Notch1 pathway was both inhibited by DAPT and ISL. The mRNA and protein expression levels of Notch1 were altered in a similar trend with cell proliferation and in a reverse trend with GFAP and $\beta$ III tubulin, indicating that Notch1 signaling was blocked directly or indirectly by ISL and therefore changed the proliferation and differentiation of GSCs. However, there was no obvious difference in Notch1 pathway expression between low dose $(20 \mu \mathrm{M})$ and high dose $(80 \mu \mathrm{M})$ ISL groups. This is possible as other pathways may be involved. In terms of differentiation, cells treated with low dose and high dose ISL had the same protein level of differentiation markers. The possible reason may be that cell differentiationinduced action reached a plateau. DAPT induced lower Notch1 and Hes1 expression levels compared to the ISL groups. It is proposed that DAPT and ISL might have different target sites of downregulation of Notch1. Further studies are still required to investigate the crosstalk between Notch and other signaling pathways, including the NF- $\mathrm{kB}$, Wnt and PI3K/Akt/mTOR pathways in GSCs.

ISL is a flavonoid compound with efficient antitumor activity. However, the drug clearance has not been measured in recent studies. Yang et al (33) firstly reported the transmembrane transport of ISL and other cardio-cerebral vascular protection flavonoids using the $\mathrm{CaCo}-2$ and blood-brain barrier (BBB) cell models in vitro. An increasing number of studies have shown that flavonoids are capable of crossing the blood-brain barrier via different BBB models such as RBE-4 cells, hCMEC/D3 cells, and ECV304/C6 coculture (34-36). In situ (rat) models have demonstrated that flavonoids are able to traverse the BBB in vivo. In recent years, new strategies such as the use of carrier-mediated transport systems and nanotechnology-based approaches enable drug transport into the brain (37). These will hopefully reduce the excretion of ISL by the body and lead toward the successful treatment of glioma patients. Nevertheless, further studies are still required to reveal the underlying mechanisms and comfirm the benefit of ISL treatment in vivo.

In conclusion, the present study confirmed our hypothesis that ISL can effectively inhibit the proliferation and induce the differentiation of human GSCs. The therapeutic effect may be related to downregulation of the Notch1 signaling pathway. However, further studies are needed to identify the target/ receptor molecules of ISL on the cell surface, and to comfirm the therapeutic effect in vivo. These studies will help to screen for the optimal parameters and elucidate the mechanism of ISL as a promising agent for the treatment of human glioma.

\section{References}

1. Chen J, McKay RM and Parada LF: Malignant glioma: Lessons from genomics, mouse models, and stem cells. Cell 149: 36-47, 2012 .

2. Lau D, Magill ST and Aghi MK: Molecularly targeted therapies for recurrent glioblastoma: Current and future targets. Neurosurg Focus 37: E15, 2014.

3. Clarke J, Penas C, Pastori C, Komotar RJ, Bregy A, Shah AH, Wahlestedt C and Ayad NG: Epigenetic pathways and glioblastoma treatment. Epigenetics 8: 785-795, 2013.

4. Codrici E, Enciu A-M, Popescu I-D, Mihai S and Tanase C: Glioma stem cells and their microenvironments: Providers of challenging therapeutic targets. Stem Cells Int 2016: 5728438, 2016. 
5. Chen J, Li Y, Yu T-S, McKay RM, Burns DK, Kernie SG and Parada LF: A restricted cell population propagates glioblastoma growth after chemotherapy. Nature 488: 522-526, 2012.

6. Schonberg DL, Lubelski D, Miller TE and Rich JN: Brain tumor stem cells: Molecular characteristics and their impact on therapy. Mol Aspects Med 39: 82-101, 2014.

7. Peng F, Du Q, Peng C, Wang N, Tang H, Xie X, Shen J and Chen J: A Review: The pharmacology of isoliquiritigenin. Phytother Res 29: 969-977, 2015.

8. Weng CJ and Yen GC: Flavonoids, a ubiquitous dietary phenolic subclass, exert extensive in vitro anti-invasive and in vivo antimetastatic activities. Cancer Metastasis Rev 31: 323-351, 2012.

9. Jung SK, Lee M-H, Lim DY, Kim JE, Singh P, Lee SY, Jeong CH, Lim TG, Chen H, Chi YI, et al: Isoliquiritigenin induces apoptosis and inhibits xenograft tumor growth of human lung cancer cells by targeting both wild type and L858R/T790M mutant EGFR. J Biol Chem 289: 35839-35848, 2014.

10. Lee SK, Park K-K, Kim KR, Kim H-J and Chung W-Y: Isoliquiritigenin inhibits metastatic breast cancer cell-induced receptor activator of nuclear factor kappa-B ligand/osteoprotegerin ratio in human osteoblastic cells. J Cancer Prev 20 281-286, 2015.

11. Wu CH, Chen HY, Wang CW, Shieh TM, Huang TC, Lin LC, Wang KL and Hsia SM: Isoliquiritigenin induces apoptosis and autophagy and inhibits endometrial cancer growth in mice. Oncotarget 7: 73432-73447, 2016.

12. Zhang X, Yeung ED, Wang J, Panzhinskiy EE, Tong C, Li W and Li J: Isoliquiritigenin, a natural anti-oxidant, selectively inhibits the proliferation of prostate cancer cells. Clin Exp Pharmacol Physiol 37: 841-847, 2010.

13. Zhou GS, Song LJ and Yang B: Isoliquiritigenin inhibits proliferation and induces apoptosis of U87 human glioma cells in vitro. Mol Med Rep 7: 531-536, 2013.

14. Platonova N, Lesma E, Basile A, Bignotto M, Garavelli S, Palano MT, Moschini A, Neri A, Colombo M and Chiaramonte R: Targeting Notch as a therapeutic approach for human malignancies. Curr Pharm Des 23: 108-134, 2017.

15. Majidinia M, Alizadeh E, Yousefi B, Akbarzadeh $M$ and Zarghami N: Downregulation of Notch signaling pathway as an effective chemosensitizer for cancer treatment. Drug Res (Stuttg) 66: 571-579, 2016.

16. Yahyanejad S, King H, Iglesias VS, Granton PV, Barbeau LM, van Hoof SJ, Groot AJ, Habets R, Prickaerts J, Chalmers AJ, et al: NOTCH blockade combined with radiation therapy and temozolomide prolongs survival of orthotopic glioblastoma. Oncotarget 7: 41251-41264, 2016.

17. Lee CK, Son SH, Park KK, Park JH, Lim SS and Chung WY: Isoliquiritigenin inhibits tumor growth and protects the kidney and liver against chemotherapy-induced toxicity in a mouse xenograft model of colon carcinoma. J Pharmacol Sci 106 444-451, 2008

18. Duan JJ, Qiu W, Xu SL, Wang B, Ye XZ, Ping YF, Zhang X, Bian XW and Yu SC: Strategies for isolating and enriching cancer stem cells: Well begun is half done. Stem Cells Dev 22: 2221-2239, 2013

19. Wu B, Sun C, Feng F, Ge M and Xia L: Do relevant markers of cancer stem cells CD133 and Nestin indicate a poor prognosis in glioma patients? A systematic review and meta-analysis. J Exp Clin Cancer Res 34: 44, 2015.

20. Zhao S, Chang H, Ma P, Gao G, Jin C, Zhao X, Zhou W and Jin B: Inhibitory effect of DNA topoisomerase inhibitor isoliquiritigenin on the growth of glioma cells. Int J Clin Exp Pathol 8: 12577-12582, 2015.
21. Wang N, Wang Z, Peng C, You J, Shen J, Han S and Chen J: Dietary compound isoliquiritigenin targets GRP78 to chemosensitize breast cancer stem cells via $\beta$-catenin/ABCG2 signaling. Carcinogenesis 35: 2544-2554, 2014

22. Wang N, Wang Z, Wang Y, Xie X, Shen J, Peng C, You J, Peng F, Tang H, Guan X, et al: Dietary compound isoliquiritigenin prevents mammary carcinogenesis by inhibiting breast cancer stem cells through WIF1 demethylation. Oncotarget 6: 9854-9876, 2015.

23. Chen H, Zhang B, Yuan X, Yao Y, Zhao H, Sun X and Zheng Q: Isoliquiritigenin-induced effects on Nrf2 mediated antioxidant defence in the HL-60 cell monocytic differentiation. Cell Biol Int 37: 1215-1224, 2013

24. Chen X, Zhang B, Yuan X, Yang F, Liu J, Zhao H, Liu L, Wang Y, Wang $Z$ and Zheng Q: Isoliquiritigenin-induced differentiation in mouse melanoma B16F0 cell line. Oxid Med Cell Longev 2012: 534934, 2012.

25. Venere M, Fine HA, Dirks PB and Rich JN: Cancer stem cells in gliomas: Identifying and understanding the apex cell in cancer's hierarchy. Glia 59: 1148-1154, 2011.

26. Singh SK, Clarke ID, Terasaki M, Bonn VE, Hawkins C, Squire J and Dirks PB: Identification of a cancer stem cell in human brain tumors. Cancer Res 63: 5821-5828, 2003.

27. Guo J, Qiang M and Ludueña RF: The distribution of $\beta$-tubulin isotypes in cultured neurons from embryonic, newborn, and adult mouse brains. Brain Res 1420: 8-18, 2011.

28. Guo J, Walss-Bass C and Ludueña RF: The beta isotypes of tubulin in neuronal differentiation. Cytoskeleton (Hoboken) 67: 431-441, 2010.

29. Gage FH and Temple S: Neural stem cells: Generating and regenerating the brain. Neuron 80: 588-601, 2013.

30. Kim MY, Mo JS, Ann EJ, Yoon JH and Park HS: Dual regulation of notch1 signaling pathway by adaptor protein fe65. J Biol Chem 287: 4690-4701, 2012

31. Purow BW, Haque RM, Noel MW, Su Q, Burdick MJ, Lee J, Sundaresan T, Pastorino S, Park JK, Mikolaenko I, et al: Expression of Notch-1 and its ligands, Delta-like-1 and Jagged-1, is critical for glioma cell survival and proliferation. Cancer Res 65: 2353-2363, 2005

32. Fan X, Matsui W, Khaki L, Stearns D, Chun J, Li YM and Eberhart CG: Notch pathway inhibition depletes stem-like cells and blocks engraftment in embryonal brain tumors. Cancer Res 66: 7445-7452,2006.

33. Yang Y, Bai L, Li X, Xiong J, Xu P, Guo C and Xue M: Transport of active flavonoids, based on cytotoxicity and lipophilicity: An evaluation using the blood-brain barrier cell and Caco-2 cell models. Toxicol In Vitro 28: 388-396, 2014.

34. Faria A, Pestana D, Teixeira D, Azevedo J, De Freitas V, Mateus N and Calhau C: Flavonoid transport across RBE4 cells: A bloodbrain barrier model. Cell Mol Biol Lett 15: 234-241, 2010.

35. Youdim KA, Qaiser MZ, Begley DJ, Rice-Evans CA and Abbott NJ: Flavonoid permeability across an in situ model of the blood-brain barrier. Free Radic Biol Med 36: 592-604, 2004.

36. Faria A, Meireles M, Fernandes I, Santos-Buelga C, GonzalezManzano S, Dueñas M, de Freitas V, Mateus N and Calhau C: Flavonoid metabolites transport across a human BBB model. Food Chem 149: 190-196, 2014.

37. Fonseca-Santos B, Gremião MP and Chorilli M: Nanotechnology-based drug delivery systems for the treatment of Alzheimer's disease. Int J Nanomedicine 10: 4981-5003, 2015. 